Check for updates

Cite this: RSC Adv., 2017, 7, 43132

\title{
Tuning analog resistive switching and plasticity in bilayer transition metal oxide based memristive synapses $\uparrow$
}

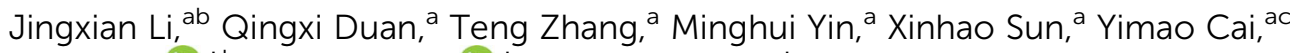 \\ Lidong Li, (D)*b Yuchao Yang (D) *ac and Ru Huang*ac
}

\begin{abstract}
Memristive devices with analog resistive switching characteristics are widely investigated nowadays for electronic synapses that facilitate memory and learning in neuromorphic computing hardware. It is therefore essential to understand and optimize the incremental switching behavior of the cells in order to enhance the functionality of memristive neural networks. Here we report a systematic study on the analog switching of bilayer oxide based memristive synapses and show that transition metal oxides with rich intermediate phases, such as $W_{x}$, are able to provide larger number of conductance states compared with oxides with few intermediate phases such as $\mathrm{TaO}_{x}$ and $\mathrm{HfO}_{x}$. This could be attributed to the intrinsically different electrical properties of the intermediate phases that jointly contribute to the change of device conductance, in addition to that caused by the varied geometry of filaments during programming. Controlled studies adopting different materials, compositions and sequences of oxide bilayers reveal that the analog switching is mainly dominated by the switching layer, thus providing clues to the optimization of memristive devices for future neuromorphic applications.
\end{abstract}

Received 8th July 2017

Accepted 31st August 2017

DOI: $10.1039 / \mathrm{c} 7 \mathrm{ra07522g}$

rsc.li/rsc-advances using memristors till now, such as spike timing dependent plasticity, ${ }^{\mathbf{1 0}}$ paired pulse facilitation, ${ }^{\mathbf{1 1}}$ heterosynaptic plasticity, ${ }^{12,13}$ etc.

It should be noted that the requirement placed on device characteristics in neuromorphic computing significantly departs from that in nonvolatile memory application, e.g. in terms of the number of states (NOS) that can be reliably accessed during resistive switching. To date, synaptic devices based on various binary transition metal oxides such as $\mathrm{TaO}_{x}{ }^{14,15} \mathrm{HfO}_{x},{ }^{16-18} \mathrm{TiO}_{x}{ }^{19-22}$ and $\mathrm{WO}_{x}{ }^{23-28}$ have been widely studied. The RS behaviors have been further engineered by introducing different interfacial layers and forming a bilayer structure. ${ }^{29-39}$ However, it is still unclear how the switching materials should be optimized in order to better fit the requirement in synaptic applications.

In this work, we have systematically studied the analog switching characteristics in memristive synapses based on bilayer oxide structures composed of a switching layer and a base layer. Through controlled investigations on the materials, compositions and stacking sequences of the oxide bilayer, it was found that the switching layer plays a dominant role in the analog switching. More importantly, the existence of rich suboxide phases in the switching layer material was found to be favorable for increasing the number of weight states, despite the fact that the intermediate oxide phases are usually deemed undesirable for nonvolatile memory applications. The richness of multilevel states could be attributed to the different electrical properties of the suboxide phases that jointly contributes to the 
change of device conductance, in addition to that caused by the varied geometry of filaments during programming. The findings in this work could shed light on the mechanism of analog switching in oxide memristors and provide clues to further optimization of memristive synapses.

\section{Experimental}

All devices studied in this work were fabricated in a $1 \times 20$ crossbar array on $\mathrm{SiO}_{2}(150 \mathrm{~nm}) / \mathrm{Si}$ substrates, as illustrated in Fig. 1a. Both the bottom and top electrodes were patterned by photo lithography, and subsequently $30 \mathrm{~nm}$ thick Pt film with
$5 \mathrm{~nm}$ thick Ti adhesion layer was deposited by e-beam evaporation and adopted as the electrodes after lift-off processes. Each cell has a size of $2 \times 2 \mu \mathrm{m}^{2}$, as shown in the scanning electron microscopy (SEM) image in Fig. 1b. We have prepared four different transition metal oxide based bilayer structures varied in stacking sequence and material/composition, namely $\mathrm{TaO}_{y} / \mathrm{Ta}_{2} \mathrm{O}_{5-x}, \mathrm{Ta}_{2} \mathrm{O}_{5-x} / \mathrm{TaO}_{y}, \mathrm{WO}_{z^{-}} 50 \% / \mathrm{TaO}_{y}$ and $\mathrm{Ta}_{2} \mathrm{O}_{5-x} / \mathrm{WO}_{z^{-}}$ $10 \%$, as the switching materials of the devices, in which the oxygen-rich $\mathrm{Ta}_{2} \mathrm{O}_{5-x}$ and $\mathrm{WO}_{z}-50 \%$ layers largely decide the overall device resistance and thus serve as the switching layers while the oxygen-deficient $\mathrm{TaO}_{y}$ and $\mathrm{WO}_{z}-10 \%$ films act as the base layers. The thicknesses of the switching layer and the base (a)

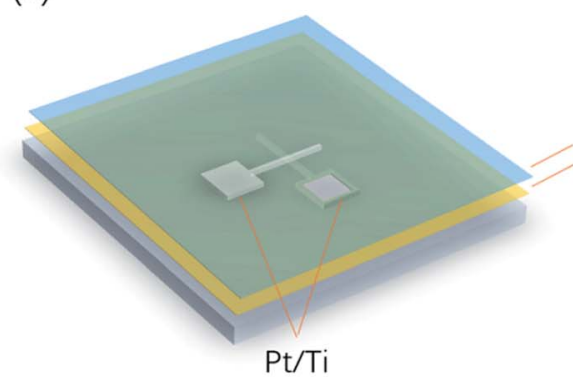

(b)

Switching layer Base layer

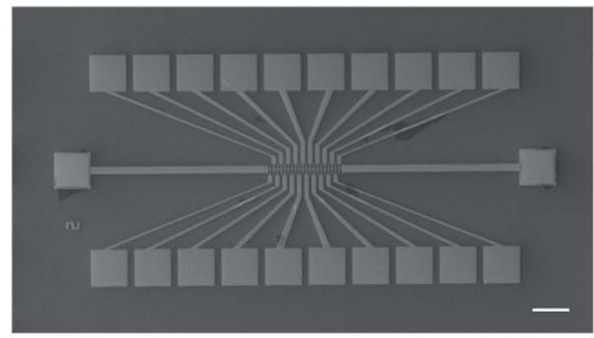

(c)

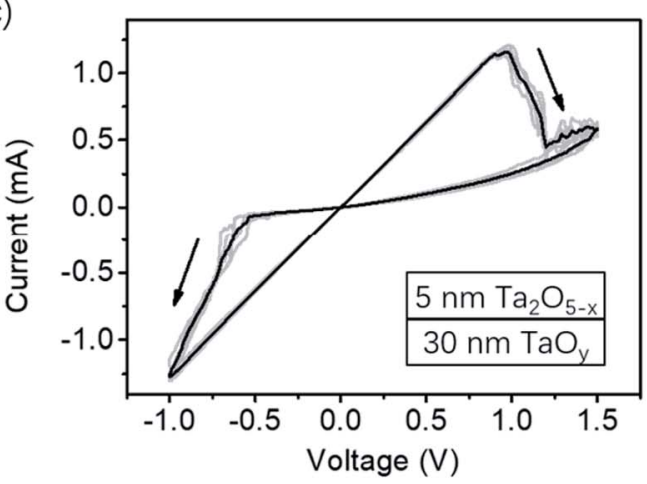

(e)

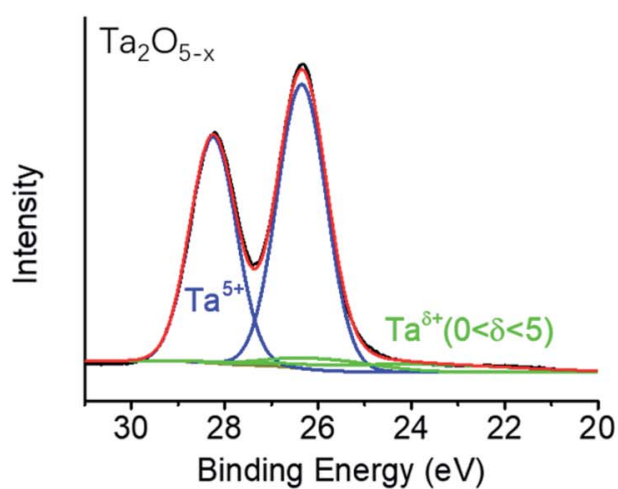

(d)

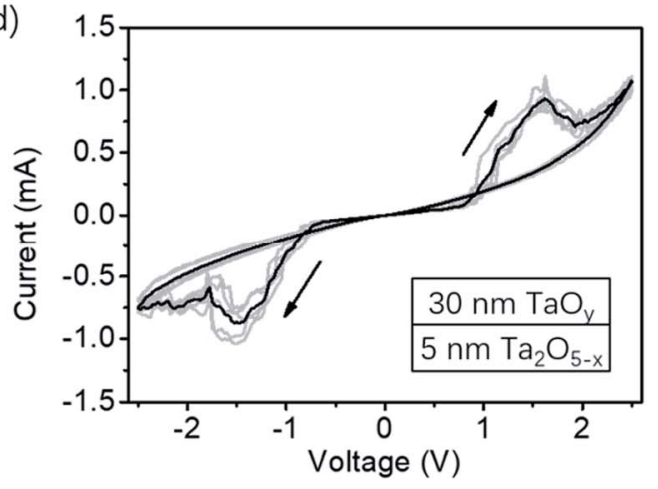

(f)

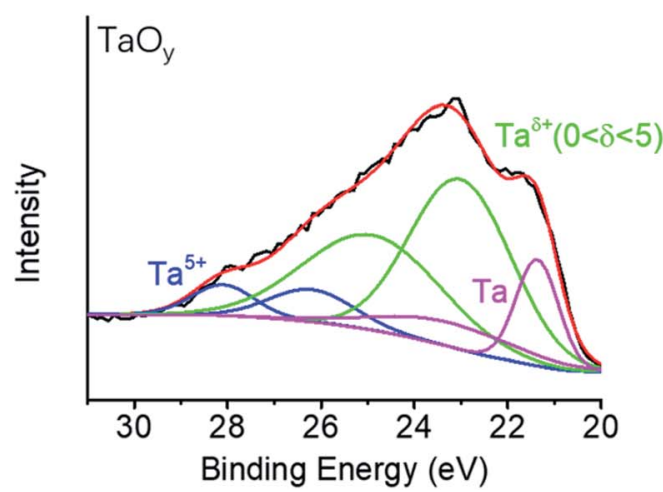

Fig. 1 (a) Schematic of the bilayer device structure. (b) SEM image of the $1 \times 20$ crossbar array. Scale bar: $100 \mu \mathrm{m}$. (c) 5 consecutive $I-V$ sweeps (gray) and averaged $I-V$ curve (black) of a Pt/Ti/ $\mathrm{Ta}_{2} \mathrm{O}_{5-x} / \mathrm{TaOy} / \mathrm{Pt}$ device. (d) 5 consecutive $I-V$ sweeps (gray) and averaged $I-V$ curve (black) of a Pt/Ti/Ta $\mathrm{O}_{5-x} / \mathrm{TaO}_{y} / \mathrm{Pt}$ device. (d) 5 consecutive $I-V$ sweeps (gray) and averaged $I-V$ curve (black) of a Pt/Ti/TaO $/ \mathrm{Ta}_{2} \mathrm{O}_{5-x} / \mathrm{Pt}$ device. (e, f) Ta $4 \mathrm{f}$ XPS spectra obtained from the blank $\mathrm{Ta}_{2} \mathrm{O}_{5-x}$ and $\mathrm{TaO}_{y}$ film, respectively. Blue, green and magenta lines correspond to $\mathrm{Ta} 4 \mathrm{f}$ doublets from the fully oxidic, suboxidic and metallic states, respectively. 
layer were always fixed to $\sim 30 \mathrm{~nm}$ and $\sim 5 \mathrm{~nm}$, respectively. The $\mathrm{Ta}_{2} \mathrm{O}_{5-x}$ layer was prepared by radio frequency (RF) sputtering of a nominally stoichiometric $\mathrm{Ta}_{2} \mathrm{O}_{5}$ target, while the $\mathrm{TaO}_{y}$ layer was deposited by direct current (DC) reactive sputtering of a Ta metal target in $\mathrm{Ar} / \mathrm{O}_{2}$ gas mixture (the oxygen partial pressure was $3 \%$ ) at $400{ }^{\circ} \mathrm{C}$ (more details can be found in our previous work $\left.{ }^{40}\right)$. The $\mathrm{WO}_{z}-50 \%$ and $\mathrm{WO}_{z}-10 \%$ films with different oxygen concentrations were deposited by reactive sputtering in $\mathrm{Ar} / \mathrm{O}_{2}$ gas mixture using a $\mathrm{W}$ metal target, and the percentage following $\mathrm{WO}_{z}$ denotes the oxygen partial pressure that was experimentally used in depositions. Electrical measurements including DC and pulse tests were performed using an Agilent B1500A semiconductor parameter analyzer together with a probe station. The electrical stimuli were always applied on the top electrodes with bottom electrodes grounded. The microstructure of the devices was analysed by high-resolution transmission electron microscopy (HRTEM) and scanning transmission electron microscopy (STEM). The compositions and chemical states of the oxides were analyzed by X-ray photoelectron spectroscopy (XPS), together with detailed peak deconvolutions. The values of $x$ and $y$ in $\mathrm{Ta}_{2} \mathrm{O}_{5-x}$ and $\mathrm{TaO}_{y}$ have been extracted by XPS, which were found to be $\sim 0.4$ and $\sim 1.0$, respectively.

\section{Results and discussion}

Fig. 1c and d show typical current-voltage $(I-V)$ characteristics of $\mathrm{Pt} / \mathrm{Ti} / \mathrm{Ta}_{2} \mathrm{O}_{5-x} / \mathrm{TaO}_{y} / \mathrm{Pt}$ and $\mathrm{Pt} / \mathrm{Ti} / \mathrm{TaO}_{y} / \mathrm{Ta}_{2} \mathrm{O}_{5-x} / \mathrm{Pt}$ devices, respectively, where the stacking sequences of the $\mathrm{Ta}_{2} \mathrm{O}_{5-x}$ and $\mathrm{TaO}_{y}$ films are opposite in these two structures. Despite the identical deposition conditions of $\mathrm{Ta}_{2} \mathrm{O}_{5-x}$ and $\mathrm{TaO}_{y}$ films in both structures, the $\mathrm{Pt} / \mathrm{Ti} / \mathrm{Ta}_{2} \mathrm{O}_{5-x} / \mathrm{TaO}_{y} / \mathrm{Pt}$ and $\mathrm{Pt} / \mathrm{Ti} / \mathrm{TaO}_{y} /$ $\mathrm{Ta}_{2} \mathrm{O}_{5-x} / \mathrm{Pt}$ devices exhibit distinct switching behaviors after electroforming. When the $\mathrm{TaO}_{y}$ film was sputtered before the $\mathrm{Ta}_{2} \mathrm{O}_{5-x}$ film, the resultant $\mathrm{Pt} / \mathrm{Ti} / \mathrm{Ta}_{2} \mathrm{O}_{5-x} / \mathrm{TaO}_{y} / \mathrm{Pt}$ device shows typical bipolar switching (Fig. 1c), which can be well explained by the $\mathrm{V}_{\mathrm{O}}$ exchange between the $\mathrm{Ta}_{2} \mathrm{O}_{5-x}$ and $\mathrm{TaO}_{y}$ layers. ${ }^{29,40,41}$ In this case, the more stoichiometric and thus more insulating $\mathrm{Ta}_{2} \mathrm{O}_{5-x}$ layer on top serves as the switching layer where local filament formation/dissolution is deemed to take place, while the more oxygen-deficient and thus more conductive $\mathrm{TaO}_{y}$ layer beneath acts as the base layer and hence can be considered as a reservoir of oxygen vacancies $\left(\mathrm{V}_{\mathrm{O}} \mathrm{S}\right)$. The migration of oxygen vacancies from the oxygen-deficient $\mathrm{TaO}_{y}$ layer to the oxygenrich $\mathrm{Ta}_{2} \mathrm{O}_{5-x}$ layer and accompanying electrochemical redox reactions lead to a set process, while backward $\mathrm{V}_{\mathrm{O}}$ migration and redox reactions result in a reset process. ${ }^{29,40,41}$ Such difference in oxygen concentration in $\mathrm{Ta}_{2} \mathrm{O}_{5-x}$ and $\mathrm{TaO}_{y}$ was clearly verified by detailed XPS analysis, as shown in Fig. $1 \mathrm{e}$ and $\mathrm{f}$. The Ta 4 f XPS spectrum of $\mathrm{Ta}_{2} \mathrm{O}_{5-x}$ films (Fig. 1e) shows a dominant Ta 4 f doublet with Ta $4 \mathrm{f}_{7 / 2}$ and $4 \mathrm{f}_{5 / 2}$ peaks located at 26.35 and $28.25 \mathrm{eV}$, respectively. These peaks are indicative of fully oxidized $\mathrm{Ta}^{5+}$ state, consistent with the property of RF sputtered films with roughly maintained stoichiometry with that of the target. In contrast, Fig. $1 \mathrm{f}$ shows the Ta 4 f XPS spectrum of $\mathrm{TaO}_{y}$ films, where three distinct chemical states of Ta can be found after peak deconvolutions. Besides the $\mathrm{Ta}^{5+}$ doublet with the Ta
$4 \mathrm{f}_{7 / 2}$ peak located at $26.2 \mathrm{eV}$, a suboxidic and metallic Ta doublets can be clearly identified with $\mathrm{Ta} 4 \mathrm{f}_{7 / 2}$ peak shifted to 23 and $21.4 \mathrm{eV} .^{40}$ Such lowered valence state of Ta clearly demonstrates the oxygen-deficient nature of the $\mathrm{TaO}_{y}$ film and therefore verifies the role of $\mathrm{TaO}_{y}$ layer as the reservoir of oxygen vacancies in the bilayer. The gradient of oxygen concentration in the bilayer structure therefore defines the polarity of the bipolar switching in $\mathrm{Pt} / \mathrm{Ti} / \mathrm{Ta}_{2} \mathrm{O}_{5-x} / \mathrm{TaO}_{y} / \mathrm{Pt}$ devices, which is favorable for enhancing the stability of cyclic switching. ${ }^{42,43}$ As a control experiment, we have also fabricated single-layer devices including $\mathrm{Pt} / \mathrm{Ti} / \mathrm{Ta}_{2} \mathrm{O}_{5-x} / \mathrm{Pt}$ and $\mathrm{Pt} / \mathrm{Ti} / \mathrm{WO}_{z}-50 \% / \mathrm{Pt}$, which have shown generally increased switching voltages and low yields, as can been in ESI Fig. S1.†

When the $\mathrm{TaO}_{y}$ film was deposited after the $\mathrm{Ta}_{2} \mathrm{O}_{5-x}$ film, however, the $\mathrm{Pt} / \mathrm{Ti} / \mathrm{TaO}_{y} / \mathrm{Ta}_{2} \mathrm{O}_{5-x} / \mathrm{Pt}$ device exhibits complementary resistive switching (CRS) behavior (Fig. 1d) that is distinct from that in Fig. 1c. CRS behavior was firstly put forward by combining two RS cells anti-serially stacked together with a common electrode. ${ }^{44}$ In this case, one cell is always in OFF state at low voltages, leading to overall high resistance and thus high nonlinearity, while the device state is defined by the internal configuration of the two cells instead of the overall resistance. Such nonlinear behavior can be used to address the sneak current problem in passive crossbar memristor arrays, ${ }^{\mathbf{4 2 , 4 4}}$ and therefore will be desirable for reducing the power consumption of memristive neural networks. These different switching effects, in oxide bilayer where only the stacking sequence was reversed, can be ascribed to interfacial reactions between the electrode and oxides. When the $\mathrm{TaO}_{y}$ film was sputtered before $\mathrm{Ta}_{2} \mathrm{O}_{5-x}$, the $\mathrm{Ta}_{2} \mathrm{O}_{5-x}$ film is in contact with the top electrode, where a $5 \mathrm{~nm}$ thick Ti adhesion layer exists between $\mathrm{Ta}_{2} \mathrm{O}_{5-x}$ and Pt. Since Ti is a well-known oxygen getter material, ${ }^{45}$ a portion of the $\mathrm{Ta}_{2} \mathrm{O}_{5-x}$ film could be reduced and thus the effective thickness of the switching layer is expected to be smaller than the nominal deposition thickness of $\mathrm{Ta}_{2} \mathrm{O}_{5-x}$, i.e. $5 \mathrm{~nm}$. On the contrary, when the $\mathrm{TaO}_{y}$ film was deposited after the $\mathrm{Ta}_{2} \mathrm{O}_{5-x}$ film, the $\mathrm{Ta}_{2} \mathrm{O}_{5-x}$ film is in immediate contact with the inert bottom Pt electrode where no Ti exists in between. As a consequence, the effective thickness of the switching layer will not be affected by the existence of $\mathrm{Ti}$ and thus is expected to be larger than that in $\mathrm{Pt} / \mathrm{Ti} / \mathrm{Ta}_{2} \mathrm{O}_{5-x} / \mathrm{TaO}_{y} / \mathrm{Pt}$ devices. Given the different effective $\mathrm{Ta}_{2} \mathrm{O}_{5-x}$ thicknesses, it is possible for the $\mathrm{V}_{\mathrm{O}} \mathrm{S}$ to get depleted in the $\mathrm{Pt} / \mathrm{Ti} / \mathrm{TaO}_{y} / \mathrm{Ta}_{2} \mathrm{O}_{5-x} / \mathrm{Pt}$ structure, leading to the observed CRS behavior in Fig. 1d rather than the bipolar switching in $\mathrm{Pt} / \mathrm{Ti} / \mathrm{Ta}_{2} \mathrm{O}_{5-x} / \mathrm{TaO}_{y} / \mathrm{Pt}$ devices (Fig. 1c). This has been verified by the reappearance of bipolar switching when a Ti layer was inserted into the bottom interface of $\mathrm{Pt} / \mathrm{Ti} / \mathrm{TaO}_{y} /$ $\mathrm{Ta}_{2} \mathrm{O}_{5-x} / \mathrm{Pt}$ devices (Fig. 2a) and also by detailed TEM characterization (Fig. 2b-e). Obviously, the $\mathrm{Ta}_{2} \mathrm{O}_{5-x}$ film when in direct contact with Ti (Fig. 2d) is thinner than the $\mathrm{Ta}_{2} \mathrm{O}_{5-x}$ without interfacial reactions with $\mathrm{Ti}$ (Fig. 2e), therefore clearly verifying our hypothesis that $\mathrm{Ti}$ has the effect of reducing a portion of $\mathrm{Ta}_{2} \mathrm{O}_{5-x}$ film and the different resistance switching behaviors in Fig. 1c and d indeed originate from interfacial reactions. As a result, a subtle variation in the thickness of the switching layer is able to result in pronounced evolutions in switching characteristics, implying that the switching layer plays a dominant 
(a)

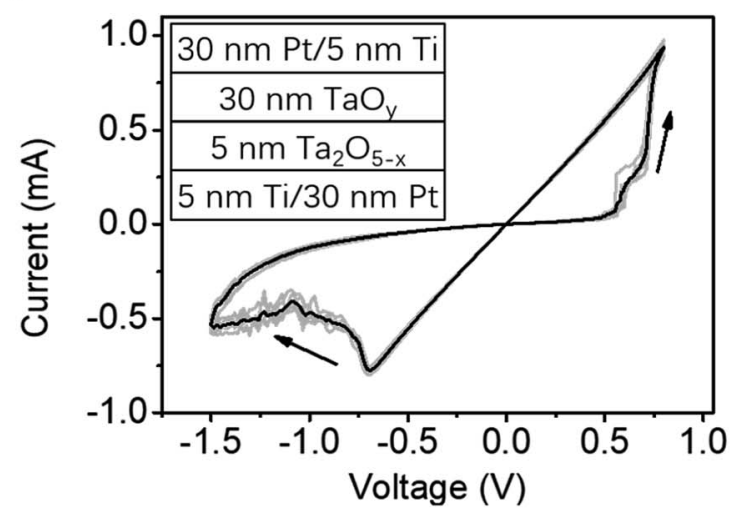

(b)

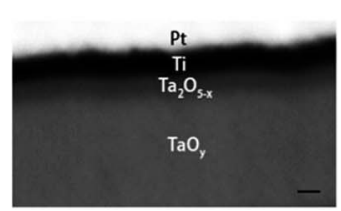

(d)

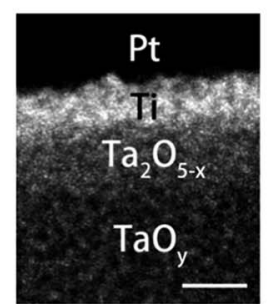

(c)

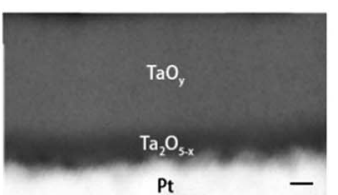

(e)

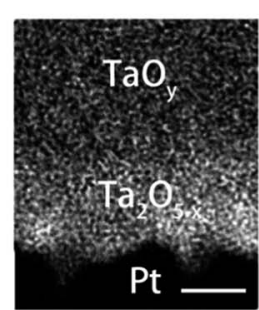

Fig. 2 (a) 5 consecutive $I-V$ sweeps (gray) and averaged $I-V$ curve (black) of a Pt/Ti/TaO $/ / \mathrm{Ta}_{2} \mathrm{O}_{5-x} / \mathrm{Ti} / \mathrm{Pt}$ device. (b and c) Cross sectional STEM images of $\mathrm{Pt} / \mathrm{Ti} / \mathrm{Ta}_{2} \mathrm{O}_{5-x} / \mathrm{TaO}_{y} / \mathrm{Ti} / \mathrm{Pt}$ and $\mathrm{Pt} / \mathrm{Ti} / \mathrm{TaO}_{y} / \mathrm{Ta}_{2} \mathrm{O}_{5-x} / \mathrm{Pt}$ devices. (d and e) Cross sectional $\mathrm{HRTEM}$ images of $\mathrm{Pt} / \mathrm{Ti} / \mathrm{Ta}_{2} \mathrm{O}_{5-x} / \mathrm{TaO}_{y} / \mathrm{Ti} / \mathrm{Pt}$ and $\mathrm{Pt} / \mathrm{Ti} / \mathrm{TaO}_{y} / \mathrm{Ta}_{2} \mathrm{O}_{5-x} / \mathrm{Pt}$ devices. Scale bar: $5 \mathrm{~nm}$.

role in resistive switching, which will be further discussed afterwards.

Furthermore, the conductance of memristors needs to be incrementally adjusted in order to serve as electronic synapses, ${ }^{12,24,46,47}$ and we have thus studied the analog conductance modulation of the aforementioned memristive devices as well. Fig. 3a shows the pulse train used for measurements on $\mathrm{Pt} / \mathrm{Ti} / \mathrm{Ta}_{2} \mathrm{O}_{5-x} / \mathrm{TaO}_{y} / \mathrm{Pt}$ devices, which contains a single, wide set pulse $\left(V_{\mathrm{SET}}=-1.1 \mathrm{~V}, t_{\mathrm{SET}}=10 \mathrm{~ms}\right)$ that ensures switching of the device to its highest conductance, followed by 50 narrower reset pulses with varied amplitudes $\left(V_{\mathrm{RESET}}=1.05-1.30 \mathrm{~V}, t_{\mathrm{RESET}}=\right.$ $200 \mathrm{~ns}, t_{\text {interval }}=10 \mathrm{~ms}$ ) aiming to incrementally reduce the device conductance and cause synaptic depression. One can see that gradual conductance reductions can be obtained as the number of reset pulses increases (Fig. 3c), and the step of conductance change upon application of each pulse $(\Delta G)$ as well as the maximum adjustment range $\left(G_{\max } / G_{\min }\right)$ can be facilely tailored by the amplitude of the applied pulses, as shown in Fig. 3c. In general, a higher $V_{\text {RESET }}$ leads to larger $\Delta G$ and $G_{\max } / G_{\min }$ (Fig. 3c), and such controllability is desirable for implementing different learning rates in memristive neural networks. Similar conductance adjustments, i.e. $\Delta G$ and $G_{\max } / G_{\min }$ dependent on the amplitude of $V_{\mathrm{SET}}$ pulses, can also be achieved in potentiation processes, as shown in Fig. $3 \mathrm{~b}$ and d. However, the conductance adjustment in potentiation (Fig. 3d) seems more abrupt compared with that in depression (Fig. 3c). This can be explained by the fact that the $\mathrm{V}_{\mathrm{O}}$ drift driven by the applied electric field and the $\mathrm{V}_{O}$ diffusion caused by the $\mathrm{V}_{\mathrm{O}}$ concentration gradient in the bilayer films are in the same direction during potentiation, i.e. both flowing from $\mathrm{TaO}_{y}$ to $\mathrm{Ta}_{2} \mathrm{O}_{5-x}$, so that the $\mathrm{V}_{\mathrm{O}}$ movement is accelerated (Fig. 3d). In stark contrast, the $\mathrm{V}_{\mathrm{O}}$ drift and diffusion are in opposite directions in depression, where reversed electric field is applied but the $\mathrm{V}_{\mathrm{O}}$ concentration gradient remains the same, therefore resulting in the overall more gradual conductance modulation observed in Fig. 3c.

Fig. 3e-h further shows the conductance evolution of $\mathrm{Pt} / \mathrm{Ti} /$ $\mathrm{TaO}_{y} / \mathrm{Ta}_{2} \mathrm{O}_{5-x} / \mathrm{Pt}$ devices when programming pulses with various amplitudes and polarities were applied. Similar with the $\mathrm{Pt} / \mathrm{Ti} / \mathrm{Ta}_{2} \mathrm{O}_{5-x} / \mathrm{TaO}_{y} / \mathrm{Pt}$ devices, the $\Delta G$ and $G_{\max } / G_{\min }$ are also strongly dependent on the amplitudes of the applied potentiation and depression pulses. Nevertheless, it can be clearly observed that the device conductance in $\mathrm{Pt} / \mathrm{Ti} / \mathrm{TaO}_{y} / \mathrm{Ta}_{2} \mathrm{O}_{5-x} / \mathrm{Pt}$ goes through a 2-regime evolution process when the pulse amplitude exceeds $-2.3 \mathrm{~V}$ (Fig. 3e and g) or $1.6 \mathrm{~V}$ (Fig. $3 \mathrm{f}$ and $\mathrm{h}$ ), where the device conductance increases first and then decreases. Such behavior is consistent with the CRS effect in Pt/ $\mathrm{Ti} / \mathrm{TaO}_{y} / \mathrm{Ta}_{2} \mathrm{O}_{5-x} / \mathrm{Pt}$ devices as observed in DC measurements and once again indicates the influence of the switching layer thickness (Fig. 1c). ${ }^{48}$

Based on the above analysis, it is obvious that the base layer and the switching layer in an oxide bilayer structure play different roles in filament growth/dissolution and resistive switching dynamics. In general, the base layer serves as a reservoir of oxygen vacancies, while the filament formation/ dissolution mainly takes place in the switching layer. This therefore indicates that the optimization of synaptic plasticity, e.g. the NOS, should be mainly performed on the material properties and defect structures of the switching layer. Tantalum oxide is a well-recognized material system with few thermodynamically stable intermediate oxide phases. ${ }^{43,49}$ As a result, the analog conductance state of the devices should mainly originate from the geometry of the conduction channels, including the length and width of the filaments. ${ }^{14}$ This to certain extent limits the NOS in tantalum oxide based memristive synapses. It is therefore of interest to replace tantalum oxide with other materials with rich intermediate phases and investigate the analog switching properties.

In light of this, we have introduced tungsten oxide as the new switching layer, which has been reported to have a large number of intermediate oxide phases including magneli phases. ${ }^{50}$ Fig. 4a shows the schematic of the $\mathrm{Pt} / \mathrm{Ti} / \mathrm{WO}_{z}-50 \% / \mathrm{TaO}_{y} / \mathrm{Pt}$ devices, where the $\mathrm{WO}_{z}-50 \%$ layer deposited using a high oxygen partial pressure of $50 \%$ in reactive sputtering was used to replace $\mathrm{Ta}_{2} \mathrm{O}_{5-x}$ and served as the switching layer while the base layer was still $\mathrm{TaO}_{y}$. The $\mathrm{W}$ 4f XPS spectrum of the 
(a)

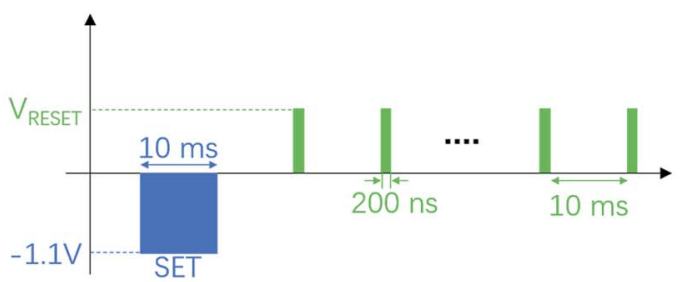

(c)

(e)
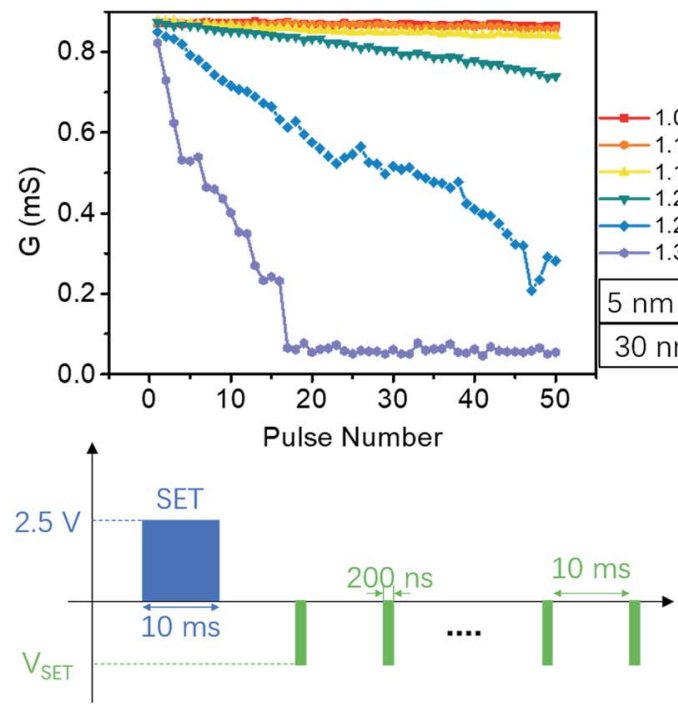

(g)

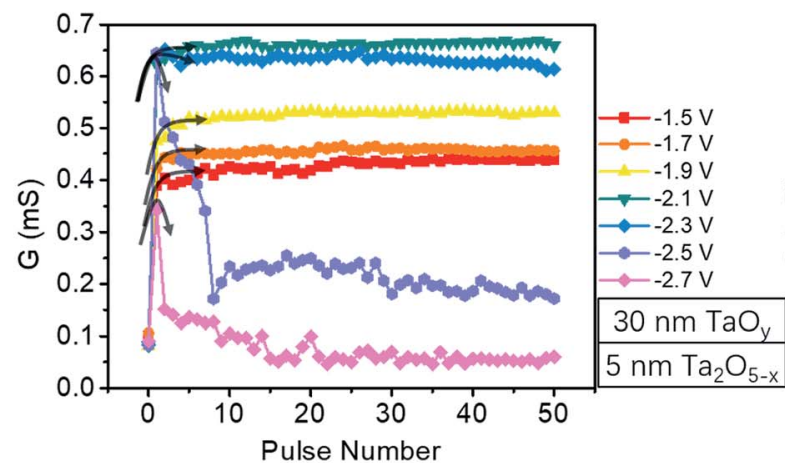

(b)

(d)

(h)
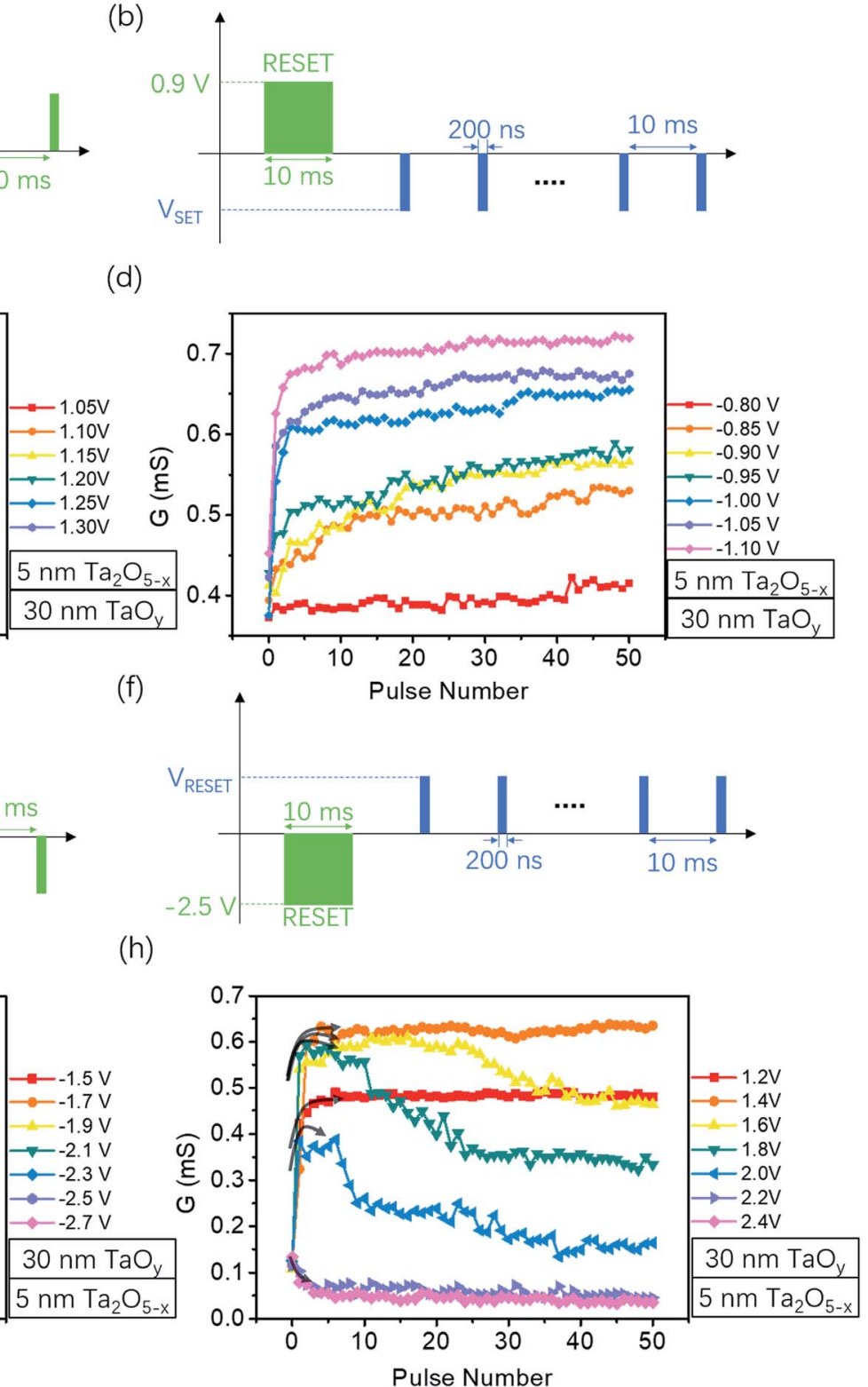

Fig. 3 (a, b) Schematic of pulse trains used for the long-term depression (LTD) and long-term potentiation (LTP) measurements with the Pt/Ti/ $\mathrm{Ta}_{2} \mathrm{O}_{5-x} / \mathrm{TaO}_{y} / \mathrm{Pt}$ device. (c, d) LTD and LTP of the Pt/Ti/Ta $\mathrm{O}_{5-x} / \mathrm{TaO}_{y} / \mathrm{Pt}$. (e, f) Schematic of pulse trains used for LTD and LTP measurements with the Pt/Ti/TaO $/ \mathrm{Ta}_{2} \mathrm{O}_{5-x} / \mathrm{Pt}$ device. (g, h) LTD and LTP of the Pt/Ti/TaO $/ / \mathrm{Ta}_{2} \mathrm{O}_{5-x} / \mathrm{Pt}$ devices.

$\mathrm{WO}_{z}-50 \%$ film (Fig. $4 \mathrm{c}$ ) shows a dominant $\mathrm{W} 4 \mathrm{f}$ doublet with $\mathrm{W}$ $4 \mathrm{f}_{7 / 2}$ and $4 \mathrm{f}_{5 / 2}$ peaks located at 36 and $38.15 \mathrm{eV}$, respectively, suggesting that the $\mathrm{WO}_{z}-50 \%$ film is nearly fully oxidized and can indeed serve as the switching layer of the device. As expected, the $\mathrm{Pt} / \mathrm{Ti} / \mathrm{WO}_{z}-50 \% / \mathrm{TaO}_{y} / \mathrm{Pt}$ devices exhibit typical bipolar switching, as shown in Fig. 4b, and the switching polarity is consistent with the built-in gradient of oxygen concentration in the $\mathrm{WO}_{z}-50 \% / \mathrm{TaO}_{y}$ bilayer. Fig. $3 \mathrm{~d}-\mathrm{g}$ further show the potentiation and depression processes in $\mathrm{Pt} / \mathrm{Ti} / \mathrm{WO}_{z^{-}}$ $50 \% / \mathrm{TaO}_{y} / \mathrm{Pt}$ devices based on pulse measurements. Compared with the conductance adjustments in $\mathrm{Pt} / \mathrm{Ti} / \mathrm{Ta}_{2} \mathrm{O}_{5-x} / \mathrm{TaO}_{y} / \mathrm{Pt}$ devices where $\mathrm{Ta}_{2} \mathrm{O}_{5-x}$ serves as the switching layer (Fig. 3c and d), it is obvious that the $\mathrm{Pt} / \mathrm{Ti} / \mathrm{WO}_{z}-50 \% / \mathrm{TaO}_{y} / \mathrm{Pt}$ devices with
$\mathrm{WO}_{z}-50 \%$ as the switching layer have shown larger NOS and better analog switching characteristics (Fig. $4 \mathrm{f}$ and g). A direct comparison of the pulse measurement results in different systems is shown in ESI Fig. S2. $\dagger$ The conductance of the Pt/Ti/ $\mathrm{WO}_{z}-50 \% / \mathrm{TaO}_{y} / \mathrm{Pt}$ devices can also be changed in a more gradual manner in both potentiation and depression processes. The $\Delta G$ and $G_{\max } / G_{\min }$ are once again dependent on the amplitudes of the potentiation and depression pulses.

The observed increase of NOS in $\mathrm{Pt} / \mathrm{Ti} / \mathrm{WO}_{z}-50 \% / \mathrm{TaO}_{y} / \mathrm{Pt}$ devices is expected to originate from the existence of rich intermediate oxide phases in tungsten oxide, as proposed above. Therefore, besides the geometric changes in conducting filaments upon application of potentiation and depression 
(a)

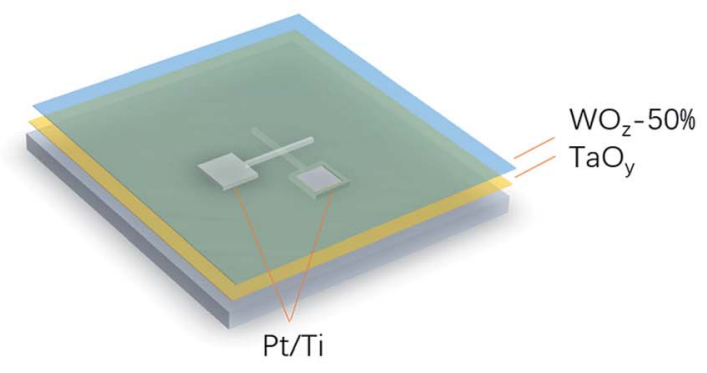

(b)

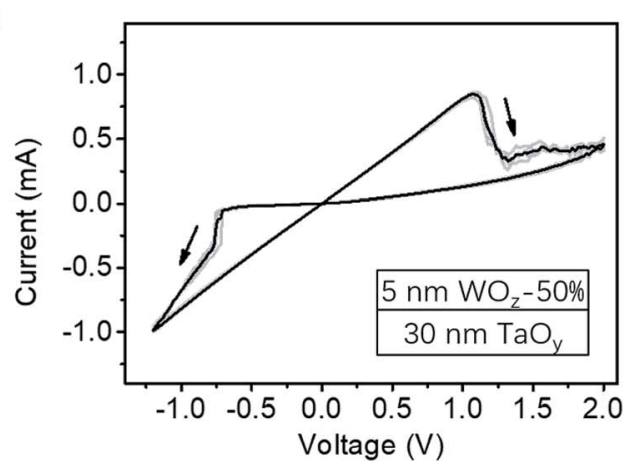

(c)

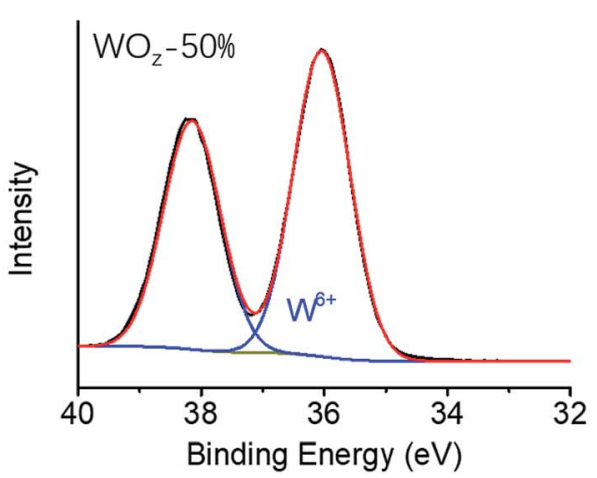

(d)

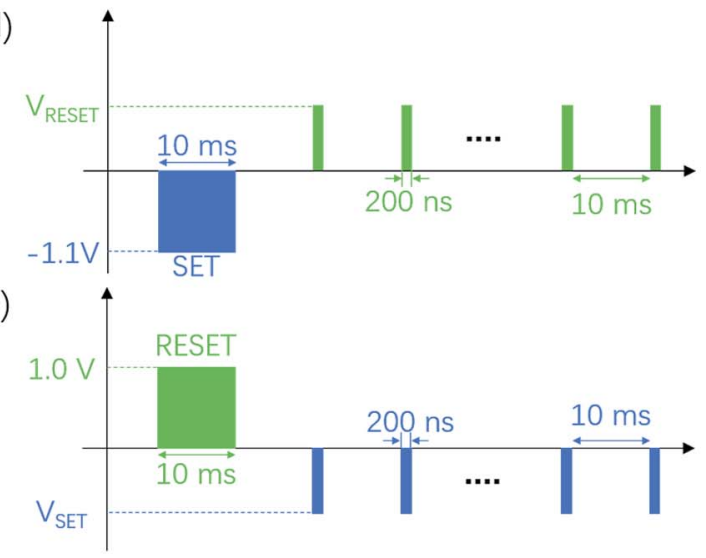

(f)

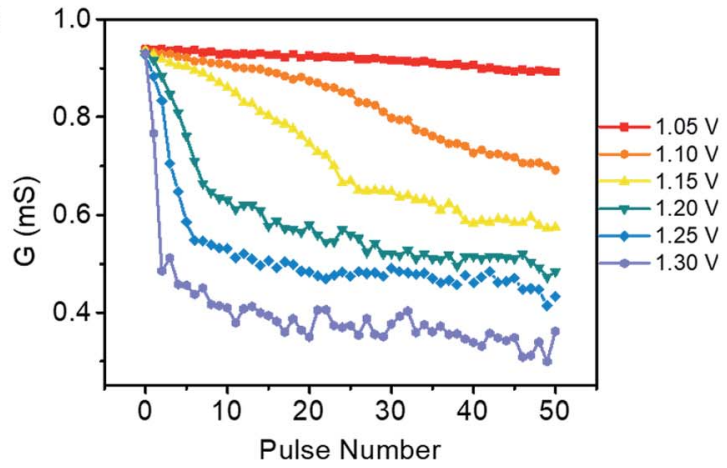

(g)

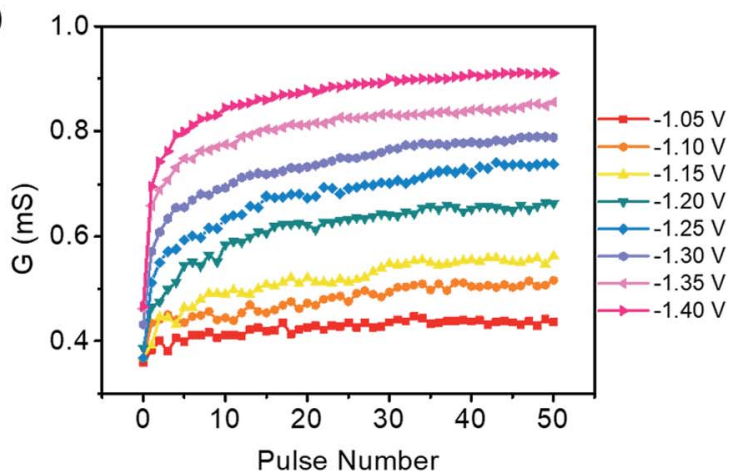

Fig. 4 (a) Schematic of the Pt/Ti/ $\mathrm{WO}_{z}-50 \% / \mathrm{TaO}_{y} / \mathrm{Pt}$ device structure. (b) 5 consecutive $/-V$ sweeps (gray) and averaged $I-V$ curve (black) of a Pt/ $\mathrm{Ti} / \mathrm{WO}_{z}-50 \% / \mathrm{TaO}_{y} / \mathrm{Pt}$ device. (c) $\mathrm{W} 4 \mathrm{f}$ XPS spectra obtained from the blank $\mathrm{WO}_{z}-50 \%$ film. Blue lines correspond to $\mathrm{W} 4 \mathrm{f}$ doublets from the fully oxidic states. (d, e) Schematic of pulse trains used for LTD and LTP measurements with the Pt/Ti/WO $-50 \% / T a O_{y} / \mathrm{Pt}$ device. (f, g) LTD and LTP of the $\mathrm{Pt} / \mathrm{Ti} / \mathrm{WO}_{z}-50 \% / \mathrm{TaO}_{y} / \mathrm{Pt}$ device.

pulses, it is possible for some localized parts of the conduction channel to undergo phase transitions between the intermediate oxide phases of $\mathrm{WO}_{z}$, further contributing to the incremental switching and thus the NOS. The applied electric field on the devices and the relatively strong thermal effects during device operations (with electrical conductance at $\sim \mathrm{mS}$ level) can provide sufficient driving force for such localized phase transitions. An example of such phase transitions in $\mathrm{WO}_{z}$ between the mother phase and a magnéli phase has been experimentally verified by transmission electron microscopy observations ${ }^{24}$ and found responsible for the resistive switching in this system. It is worthwhile noting that in the case of analog switching the conducting filament does not have to undergo phase transitions as a whole. Instead, only localized phase transitions in parts of the conduction channel will suffice in adjusting the device conductance in an incremental way, leading to the fine analog switching observed in Fig. $4 \mathrm{f}$ and $\mathrm{g}$ when the phase transition processes are coupled with the geometric changes of conducting filaments.

In order to verify this hypothesis, we have also fabricated control devices where tungsten oxide was used as the base layer instead of the switching layer (Fig. 5a). The oxygen partial pressure was reduced to $10 \%$ during the deposition of $\mathrm{WO}_{z}$ in order to increase the concentration of oxygen vacancies, 
(a)

(b)

(c)
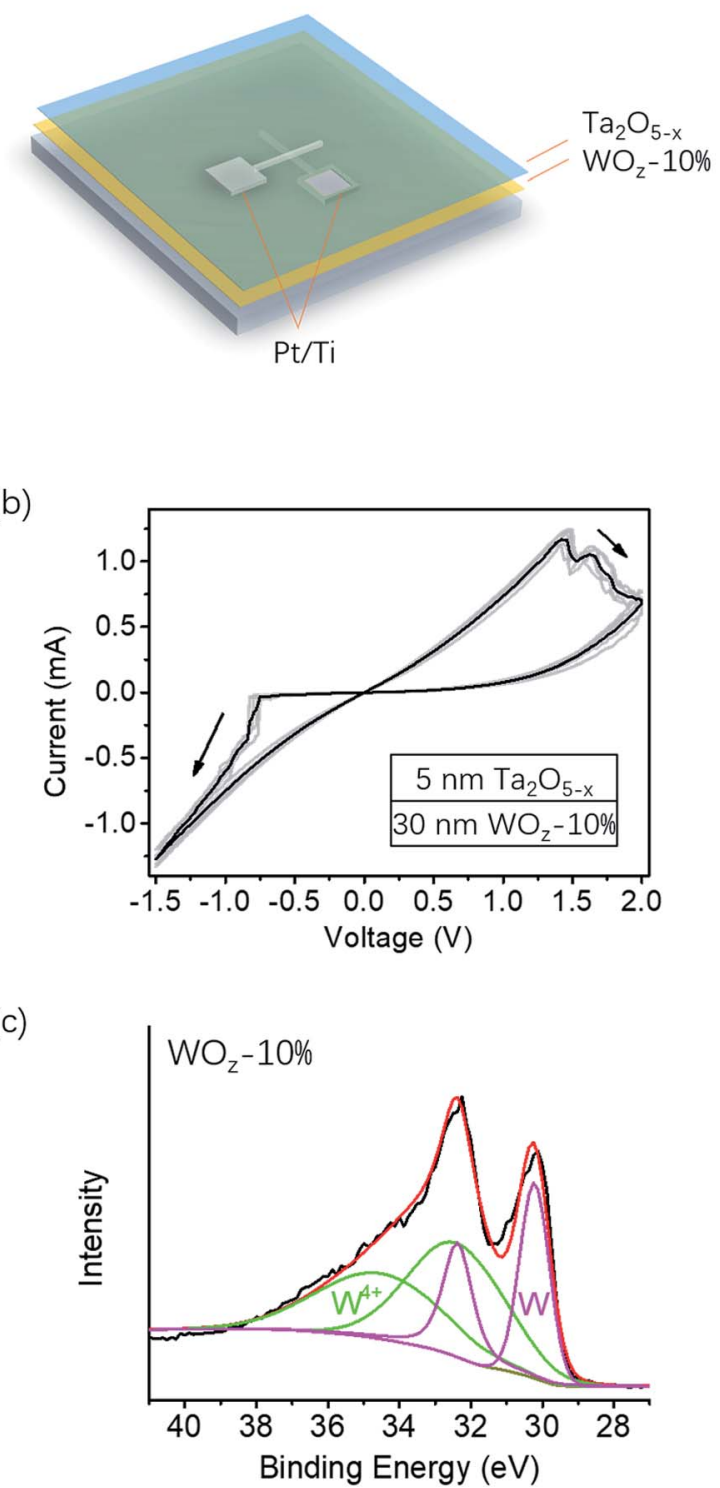

(d)

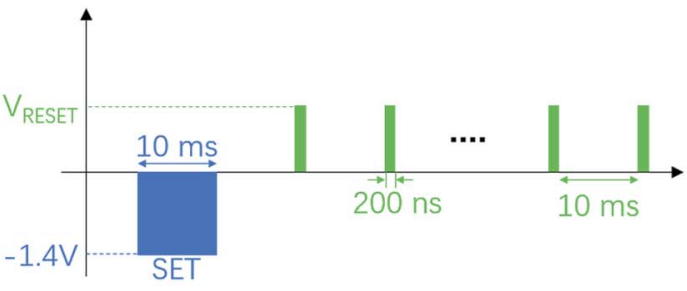

(e)

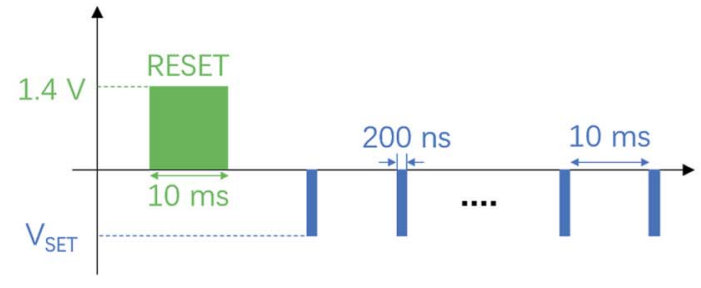

(f)

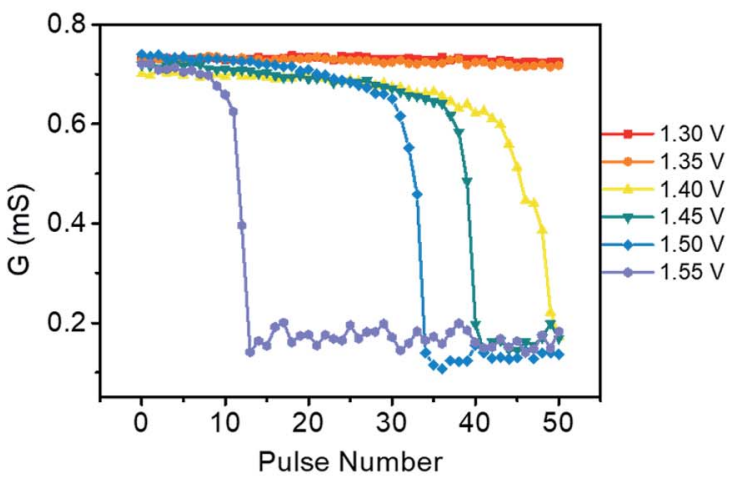

(g)

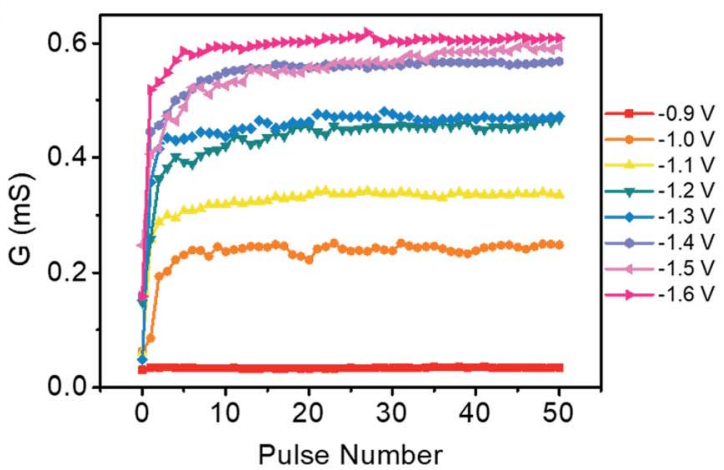

Fig. 5 (a) Schematic of the Pt/Ta $\mathrm{O}_{5-x} / \mathrm{WO}_{z}-10 \% / \mathrm{Pt}$ device structure. (b) 5 consecutive $I-V$ sweeps (gray) and averaged $I-V$ curve (black) of a Pt/ $\mathrm{Ti} / \mathrm{Ta}_{2} \mathrm{O}_{5-x} / \mathrm{WO}_{z}-10 \% / \mathrm{Pt}$ device. (c) W $4 \mathrm{f}$ XPS spectra obtained from the blank $\mathrm{WO}_{z}-10 \%$ film. Green and magenta lines correspond to $\mathrm{W} 4 \mathrm{f}$ doublets from the suboxidic and metallic states, respectively. (d, e) Schematic of pulse trains used for LTD and LTP measurements with the Pt/Ti/ $\mathrm{Ta}_{2} \mathrm{O}_{5-x} / \mathrm{WO}_{z}-10 \% / \mathrm{Pt}$ device. (f, g) LTD and LTP of the Pt/Ti/Ta $\mathrm{O}_{5-x} / \mathrm{WO}_{z}-10 \% / \mathrm{Pt}$.

therefore forming a $\mathrm{Pt} / \mathrm{Ti} / \mathrm{Ta}_{2} \mathrm{O}_{5-x} / \mathrm{WO}_{z}-10 \% / \mathrm{Pt}$ structure. The XPS spectrum from the $\mathrm{WO}_{z}-10 \%$ film clearly shows existence of reduced $\mathrm{W}$ and metallic $\mathrm{W}$ states in the film (green and magenta doublets) and hence increased $\mathrm{V}_{\mathrm{O}}$ concentration in the film, as shown in Fig. 5c. A typical oxide bilayer is thus formed when the more oxygen-deficient $\mathrm{WO}_{z}$ film is stacked with oxygen-rich $\mathrm{Ta}_{2} \mathrm{O}_{5-x}$, and the devices once again display typical bipolar switching characteristics (Fig. 5b). However, since the $\mathrm{WO}_{z}$ was used as the base layer instead of the switching layer in the present $\mathrm{Pt} / \mathrm{Ti}^{-} \mathrm{Ta}_{2} \mathrm{O}_{5-x} / \mathrm{WO}_{z}-10 \% / \mathrm{Pt}$ structure, the switching characteristics are now mainly decided by $\mathrm{Ta}_{2} \mathrm{O}_{5-x}$ and thus the intermediate states in $\mathrm{WO}_{z}$ do not effectively contribute to the analog switching, unlike in $\mathrm{Pt} / \mathrm{Ti} / \mathrm{WO}_{z}-50 \% / \mathrm{TaO}_{y} / \mathrm{Pt}$ devices. As a result, the switching of the $\mathrm{Pt} / \mathrm{Ti} / \mathrm{Ta}_{2} \mathrm{O}_{5-x} / \mathrm{WO}_{z}-10 \% / \mathrm{Pt}$ devices becomes very abrupt and the NOS largely decreases, as shown in Fig. $5 \mathrm{~d}-\mathrm{g}$, which strongly demonstrates that the incremental switching behavior and the NOS are indeed correlated with the number of intermediate oxide phases in the switching layer.

We have further examined the above hypothesis in other transition metal oxide systems, and Fig. 6a summarizes the reported NOS values in memristive synapses based on four different transition metal oxides that have been reported 
(a)

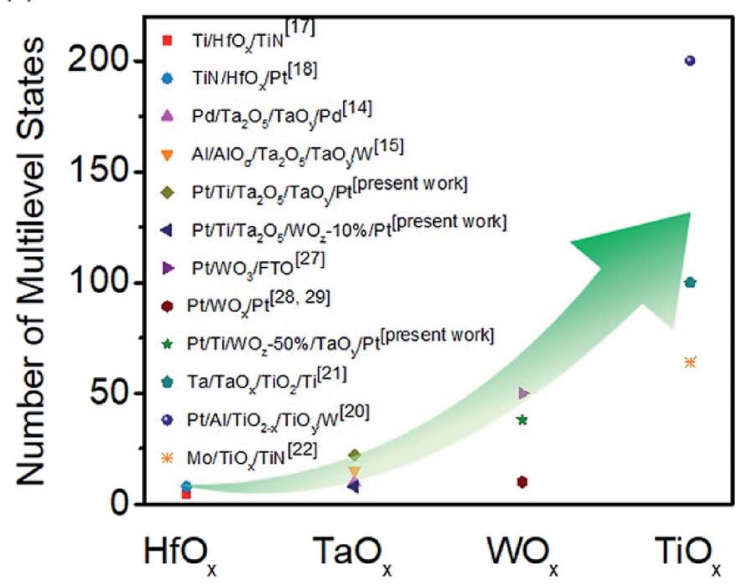

(b)

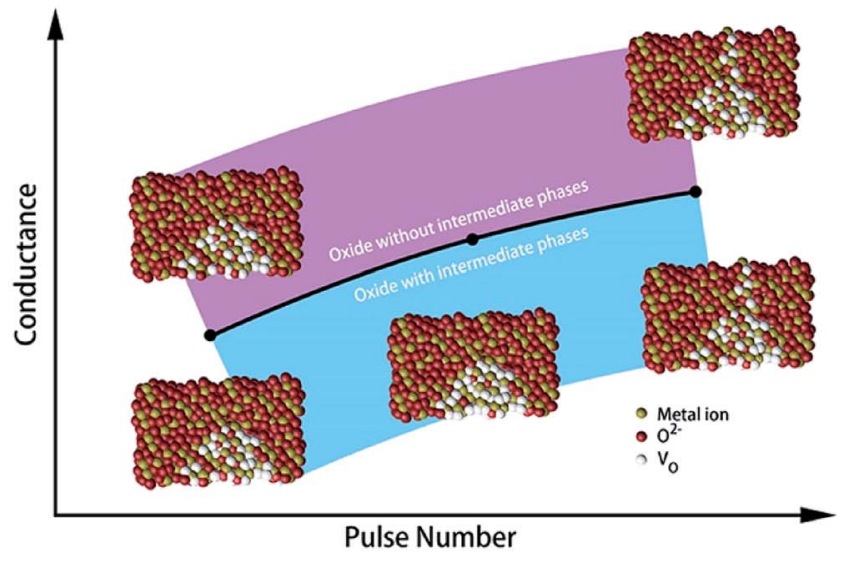

Fig. 6 (a) The number of multilevel states in four transition metal oxides based memristive synapses. (b) Schematic of the conductance change mechanism in oxide systems with and without intermediate phases, leading to different NOS.

previously, ${ }^{14,15,17,18,20-22,27-29}$ where $\mathrm{HfO}_{x}$ and $\mathrm{TaO}_{x}$ are typical material systems with few thermodynamically stable oxide phases while $\mathrm{WO}_{x}$ and $\mathrm{TiO}_{x}$ represent material systems with rich intermediate phases. ${ }^{\mathbf{4 0 , 4 3 , 4 9 - 5 2}}$ The extraction of NOS should takes into account the noise level and conductance change that could be recognized (ESI Table $\mathrm{S} 1$ and related discussions $\dagger$ ). It is obvious in Fig. 6a that $\mathrm{WO}_{x}$ and $\mathrm{TiO}_{x}$ based synaptic devices have generally shown much higher NOS compared with $\mathrm{HfO}_{x}$ and $\mathrm{TaO}_{x}$, once again verifying that there is indeed an underlying correlation between the NOS and the number of phases in oxide memristors. This correlation is schematically depicted in Fig. $6 \mathrm{~b}$, where the existence of intermediate phases provides more conductance levels when coupled with the geometric changes of filaments, compared with oxides with few thermodynamically stable intermediate phases.

We would like to finally note that here we have concentrated on tailoring the number of conductance states, which is an important criterion for memristive synapses, but other characteristics of the cells such as endurance, retention etc. will put additional constraints on the synaptic devices and sometimes have different requirements on material properties. It is also likely to have some extent of intermixing between the switching layer and the base layer, due to inevitable inter-diffusion, as verified by XPS depth profiling analysis (ESI Fig. S3†). The present findings, however, could provide important clues to the optimization of memristive cells for future neuromorphic computing applications.

\section{Conclusions}

In summary, we have systematically studied the influence of materials, compositions and stacking sequences of oxide bilayer on the analog switching characteristics of memristive synapses. The results unambiguously showed the dominant role of the switching layer in deciding the analog switching properties and therefore device optimizations should be concentrated on the switching layer. Furthermore, compared with oxides with few thermodynamically stable intermediate phases, it was found that the existence of rich intermediate phases could lead to larger NOS and thus more gradual resistive switching. These findings could be important for future device optimizations and constructions of neuromorphic computing systems.

\section{Conflicts of interest}

There are no conflicts of interest to declare.

\section{Acknowledgements}

This work was supported by National Key R\&D Program of China (2017YFA0207600), Beijing Municipal Science \& Technology Commission Program (Z161100000216148) and National Natural Science Foundation of China (61674006, 61421005). Y. Y. acknowledges support from the "1000 Youth Talents Program" of China.

\section{References}

1 L. O. Chua, IEEE Trans. Circuit Theory, 1971, 18, 507-519.

2 D. B. Strukov, G. S. Snider, D. R. Stewart and R. S. Williams, Nature, 2008, 453, 80-83.

3 J. J. Yang, D. B. Strukov and D. R. Stewart, Nat. Nanotechnol., 2013, 8, 13-24.

4 R. Waser, R. Dittmann, G. Staikov and K. Szot, Adv. Mater., 2009, 21, 2632-2663.

5 R. Waser and M. Aono, Nat. Mater., 2007, 6, 833-840.

6 Y. Yang, P. Gao, S. Gaba, T. Chang, X. Pan and W. Lu, Nat. Commun., 2012, 3, 732.

7 Y. Yang, P. Gao, L. Li, X. Pan, S. Tappertzhofen, S. Choi, R. Waser, I. Valov and W. D. Lu, Nat. Commun., 2014, 5, 4232.

8 N. K. Upadhyay, S. Joshi and J. J. Yang, Sci. China. Inform. Sci., 2016, 59, 061404.

9 M. Prezioso, F. Merrikh-Bayat, B. D. Hoskins, G. C. Adam, K. K. Likharev and D. B. Strukov, Nature, 2015, 521, 61-64. 
10 S. H. Jo, T. Chang, I. Ebong, B. B. Bhadviya, P. Mazumder and W. Lu, Nano Lett., 2010, 10, 1297-1301.

11 C. Du, W. Ma, T. Chang, P. Sheridan and W. D. Lu, Adv. Funct. Mater., 2015, 25, 4290-4299.

12 Y. Yang, B. Chen and W. D. Lu, Adv. Mater., 2015, 27, 77207727.

13 Y. Yang, M. Yin, Z. Yu, Z. Wang, T. Zhang, Y. Cai, W. D. Lu and R. Huang, Adv. Electron. Mater., 2017, 3, 1700032.

14 S. Kim, C. Du, P. Sheridan, W. Ma, S. Choi and W. D. Lu, Nano Lett., 2015, 15, 2203-2211.

15 X. Li, H. Wu, G. Bin, W. Wu, D. Wu, N. Deng, J. Cai and H. Qian, Nanotechnology, 2016, 27, 305201.

16 B. Gao, Y. J. Bi, H. Y. Chen, R. Liu, P. Huang, B. Chen, L. F. Liu, X. Y. Liu, S. M. Yu, H. S. P. Wong and J. F. Kang, ACS Nano, 2014, 8, 6998-7004.

17 Y.-E. Syu, T.-C. Chang, J.-H. Lou, T.-M. Tsai, K.-C. Chang, M.-J. Tsai, Y.-L. Wang, M. Liu and S. M. Sze, Appl. Phys. Lett., 2013, 102, 172903.

18 L. Zhao, H. Y. Chen, S. C. Wu, Z. Jiang, S. Yu, T. H. Hou, H. S. Wong and Y. Nishi, Nanoscale, 2014, 6, 5698-5702.

19 A. Fabien, G. Ligang, D. H. Brian and B. S. Dmitri, Nanotechnology, 2012, 23, 075201.

20 K. Seo, I. Kim, S. Jung, M. Jo, S. Park, J. Park, J. Shin, K. P. Biju, J. Kong, K. Lee, B. Lee and H. Hwang, Nanotechnology, 2011, 22, 254023.

21 L. Gao, I. T. Wang, P. Y. Chen, S. Vrudhula, J. S. Seo, Y. Cao, T. H. Hou and S. Yu, Nanotechnology, 2015, 26, 455204.

22 J. Park, M. Kwak, K. Moon, J. Woo, D. Lee and H. Hwang, IEEE Electron Device Lett., 2016, 37, 1559-1562.

23 T. Chang, S.-H. Jo and W. Lu, ACS Nano, 2011, 5, 7669-7676.

24 Z. H. Tan, R. Yang, K. Terabe, X. B. Yin, X. D. Zhang and X. Guo, Adv. Mater., 2016, 28, 377-384.

25 R. Yang, K. Terabe, G. Liu, T. Tsuruoka, T. Hasegawa, J. K. Gimzewski and M. Aono, ACS Nano, 2012, 6, 9515-9521.

26 T. Shi, X. B. Yin, R. Yang and X. Guo, Phys. Chem. Chem. Phys., 2016, 18, 9338-9343.

27 D. Kuzum, S. Yu and H. S. Wong, Nanotechnology, 2013, 24, 382001.

28 R. Yang, K. Terabe, Y. P. Yao, T. Tsuruoka, T. Hasegawa, J. K. Gimzewski and M. Aono, Nanotechnology, 2013, 24, 384003.

29 M.-J. Lee, C. B. Lee, D. Lee, S. R. Lee, M. Chang, J. H. Hur, Y.-B. Kim, C.-J. Kim, D. H. Seo, S. Seo, U. I. Chung, I.-K. Yoo and K. Kim, Nat. Mater., 2011, 10, 625-630.

30 S. H. Chang, S. B. Lee, D. Y. Jeon, S. J. Park, G. T. Kim, S. M. Yang, S. C. Chae, H. K. Yoo, B. S. Kang, M. J. Lee and T. W. Noh, Adv. Mater., 2011, 23, 4063-4067.

31 X. Liu, S. M. Sadaf, M. Son, J. Shin, J. Park, J. Lee, S. Park and H. Hwang, Nanotechnology, 2011, 22, 475702.
32 J. Lee, C. Du, K. Sun, E. Kioupakis and W. D. Lu, ACS Nano, 2016, 10, 3571-3579.

33 J. J. Yang, J. Borghetti, D. Murphy, D. R. Stewart and R. S. Williams, Adv. Mater., 2009, 21, 3754-3758.

34 M.-K. Kim and J.-S. Lee, ACS Appl. Mater. Interfaces, 2016, 8, 32918-32924.

35 S.-M. Lin, J.-Y. Tseng, T.-Y. Su, Y.-C. Shih, J.-S. Huang, C.-H. Huang, S.-J. Lin and Y.-L. Chueh, ACS Appl. Mater. Interfaces, 2014, 6, 17686-17693.

36 P. Bousoulas, P. Asenov, I. Karageorgiou, D. Sakellaropoulos, S. Stathopoulos and D. Tsoukalas, J. Appl. Phys., 2016, 120, 154501.

37 R. Huang, X. Yan, K. A. Morgan, M. D. B. Charlton and C. H. de Groot, J. Phys. D: Appl. Phys., 2017, 50, 175101.

38 G. Niu, M. A. Schubert, S. U. Sharath, P. Zaumseil, S. Vogel, C. Wenger, E. Hildebrandt, S. Bhupathi, E. Perez, L. Alff, M. Lehmann, T. Schroeder and T. Niermann, Nanotechnology, 2017, 28, 215702.

39 K. Park and J.-S. Lee, RSC Adv., 2016, 6, 21736-21741.

40 Y. C. Yang, S. Choi and W. Lu, Nano Lett., 2013, 13, 29082915.

41 Y. C. Yang, J. Lee, S. Lee, C. H. Liu, Z. H. Zhong and W. Lu, Adv. Mater., 2014, 26, 3693-3699.

42 Y. C. Yang, P. Sheridan and W. Lu, Appl. Phys. Lett., 2012, 100, 203112.

43 J. J. Yang, M. X. Zhang, J. P. Strachan, F. Miao, M. D. Pickett, R. D. Kelley, G. Medeiros-Ribeiro and R. S. Williams, Appl. Phys. Lett., 2010, 97, 232102.

44 E. Linn, R. Rosezin, C. Kugeler and R. Waser, Nat. Mater., 2010, 9, 403-406.

45 J. J. Yang, J. P. Strachan, F. Miao, M. X. Zhang, M. D. Pickett, W. Yi, D. A. A. Ohlberg, G. Medeiros-Ribeiro and R. S. Williams, Appl. Phys. A: Mater. Sci. Process., 2011, 102, 785-789.

46 Z. Wang, S. Joshi, S. E. Savel'ev, H. Jiang, R. Midya, P. Lin, M. $\mathrm{Hu}$, N. Ge, J. P. Strachan, Z. Li, Q. Wu, M. Barnell, G. L. Li, H. L. Xin, R. S. Williams, Q. Xia and J. J. Yang, Nat. Mater., 2017, 16, 101-108.

47 T. Ohno, T. Hasegawa, T. Tsuruoka, K. Terabe, J. K. Gimzewski and M. Aono, Nat. Mater., 2011, 10, 591-595. 48 X. Zhu, C. Du, Y. Jeong and W. D. Lu, Nanoscale, 2017, 9, 4551.

49 S. P. Garg, N. Krishnamurthy, A. Awasthi and M. Venkatraman, J. Phase Equilib., 1997, 18, 407.

50 M. Katoh and Y. Takeda, Jpn. J. Appl. Phys., Part 1, 2004, 43, 7292-7295.

51 H. Okamoto, J. Phase Equilib. Diffus., 2008, 29, 124.

52 P. G. Wahlbeck and P. W. Gilles, J. Am. Ceram. Soc., 1966, 49, 180-183. 